\title{
Selecting the Best Supervised Learning Algorithm for Recommending the Course in E-Learning System
}

\author{
Sunita B Aher \\ M.E. (CSE) -II \\ Walchand Institute of Technology \\ Solapur University India
}

\author{
Lobo L.M.R.J. \\ Associate Professor, Head, Department of IT \\ Walchand Institute of Technology \\ Solapur University India
}

\begin{abstract}
Now a day E-learning is becoming popular as it helps to fulfil the necessities of remote students and helps the teaching-learning process in Education system. Course Recommender System in E-Learning is a system which recommend the course to the student based on the choice of various student collected from huge amount of data of courses offered through Moodle package of the college. Here in this paper we compare the seven classification algorithm to choose the best classification algorithm for Course Recommendation system. Theses seven classification algorithms are ADTree, Simple Cart, J48, ZeroR, Naive Bays, Decision Table \& Random Forest Classification Algorithm. We compare these seven algorithms using open source data mining tool Weka \& present the result. We found that ADTree classification algorithm works better for this Course Recommender System than other five classification algorithms.
\end{abstract}

\section{Keywords}

ADTree, Simple Cart, J48, ZeroR, Naive Bays, Decision Table, Random Forest Classification Algorithm, Weka

\section{INTRODUCTION}

Course Recommender System in E-Learning is a system which recommend the course to the student based on the choice of various student collected from huge amount of data of courses offered through Moodle package of the college e.g. If student is interested in course like Database System then he would like to learn the Advanced Database System.

Here we use Moodle for data collection \& Weka to check the results. The data flow diagram for Course Recommender System is given in figure $1 \&$ explained in [15].

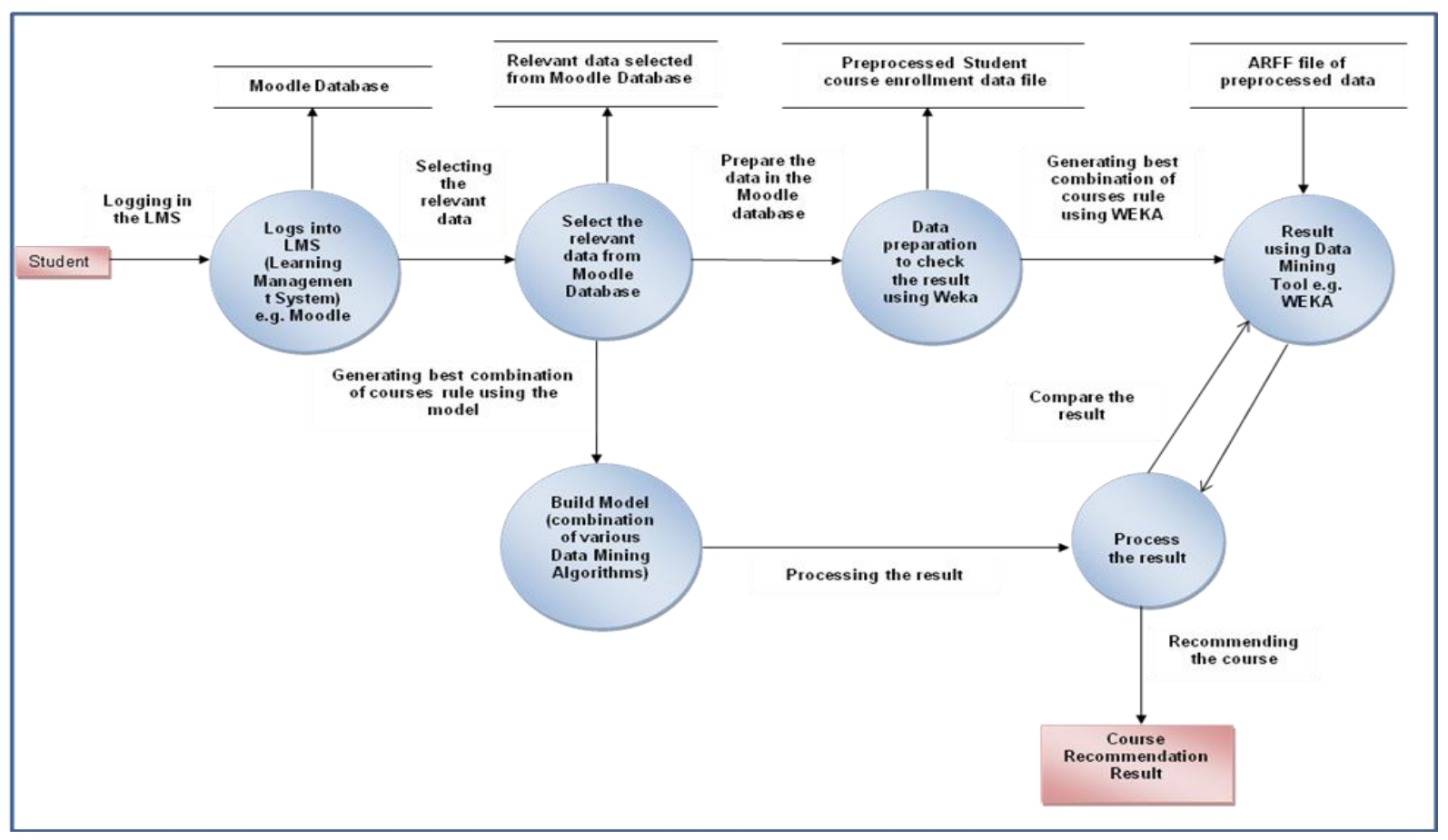

Figure 1: Data Flow Diagram for Course Recommendation System [15] 


\section{LITERATURE REVIEW}

Hu H., Li J., Plank A., Wang H. and Daggard G. [1] conducted experimental comparison of LibSVMs, C4.5, BaggingC4.5, AdaBoostingC4.5, and Random Forest on seven Microarray cancer data sets. The experimental results showed that all ensemble methods outperformed C4.5. The experimental results also showed that all five methods benefited from data preprocessing, including gene selection and discretization, in classification accuracy. In addition to comparing the average accuracies of ten-fold cross validation tests on seven data sets, they used two statistical tests to validate findings.

Abdelghani Bellaachia, Erhan Guven [2] presented an analysis of the prediction of survivability rate of breast cancer patients using data mining techniques. The data used was the SEER Public-Use Data. The preprocessed data set consists of 151,886 records, which had all the available 16 fields from the SEER database. They have investigated three data mining techniques: the Naïve Bayes, the back-propagated neural network, and the C4.5 decision tree algorithms. Several experiments were conducted using these algorithms. The achieved prediction performances were comparable to existing techniques. However, they found out that $\mathrm{C} 4.5$ algorithm has a much better performance than the other two techniques.

My Chau Tu, Dongil Shin, Dongkyoo Shin [3] proposed the use of decision tree $\mathrm{C} 4.5$ algorithm, bagging with decision tree C4.5 algorithm and bagging with Naïve Bayes algorithm to identify the heart disease of a patient and compare the effectiveness, correction rate among them. The data they studied was collected from patients with coronary artery disease.

Aman Kumar Sharma, Suruchi Sahni [4] conducted experiment in the WEKA environment by using four algorithms namely ID3, J48, Simple CART and Alternating Decision Tree on the spam email dataset and later the four algorithms were compared in terms of classification accuracy. According to their simulation results, the J48 classifier outperforms the ID3, CART and ADTree in terms of classification accuracy.

Rich Caruana Alexandru Niculescu-Mizil [5] presented a large-scale empirical comparison between ten supervised learning methods: SVMs, neural nets, logistic regression, naive bayes, memory-based learning, random forests, decision trees, bagged trees, boosted trees, and boosted stumps. They also examined the effect that calibrating the models via Platt Scaling and Isotonic Regression has on their performance. An important aspect of their study was the use of a variety of performance criteria to evaluate the learning methods.

Eric Bauer, Ron Kohavi [6] provided a brief review of two families of voting algorithms: perturb and combine (e.g., Bagging), and boosting (e.g., AdaBoost, Arc-x4).

In research [7], twenty-two decision tree, nine statistical, and two neural network algorithms were compared on thirty-two datasets in terms of classification accuracy, training time, and (in the case of trees) number of leaves. Classification accuracy is measured by mean error rate and mean rank of error rate. Both criteria place a statistical, spline-based, algorithm called Polyclass at the top, although it is not statistically significantly different from twenty other algorithms. Another statistical algorithm, logistic regression, is second with respect to the two accuracy criteria. The most accurate decision tree algorithm is Quest with linear splits, which ranks fourth and fifth, respectively. Although spline-based statistical algorithms tend to have good accuracy, they also require relatively long training times. Polyclass, for example, is third last in terms of median training time. It often requires hours of training compared to seconds for other algorithms. The Quest and logistic regression algorithms are substantially faster. Among decision tree algorithms with univariate splits, C4.5, Ind-Cart, and Quest have the best combinations of error rate and speed. But $\mathrm{C} 4.5$ tends to produce trees with twice as many leaves as those from IndCart and Quest.

\section{SUPERVISED CLASSIFICATION ALGORITHMS}

Classification is a data mining task that maps the data into predefined groups \& classes. It is also called as supervised learning. It consists of two steps. First step is the model construction which consists of set of predetermined classes. Each tuple /sample is assumed to belong to a predefined class. The set of tuple used for model construction is training set. The model is represented as classification rules, decision trees, or mathematical formulae. Second step is model usage which is used for classifying future or unknown objects. The known label of test sample is compared with the classified result from the model. Accuracy rate is the percentage of test set samples that are correctly classified by the model. Test set is independent of training set, otherwise over-fitting will occur [10]. Here we consider the brief introduction of each classification algorithm.

\subsection{ADTree Classification Algorithm}

An alternating decision tree (ADTree) is a machine learning method for classification which generalizes decision trees. An alternating decision tree consists of two nodes. Decision nodes specify a predicate condition. Prediction nodes contain a single number. ADTree always have prediction nodes as both root and leaves. An instance is classified by an ADTree by following all paths for which all decision nodes are true and summing any prediction nodes that are traversed.

The fundamental element of the ADTree algorithm is the rule as it comes under the tree category. A single rule consists of [16]:

- Condition: is a predicate of the form "attribute <comparison> value.

- Precondition: is simply a logical conjunction of conditions and

- Two scores.

A precondition Evaluation of a rule involves a pair of nested if statements:

$$
\begin{aligned}
& \begin{array}{l}
\text { if(precondition) } \\
\text { if(condition) } \\
\text { return score_one }
\end{array} \\
& \text { else return score_minus_one } \\
& \text { end if } \\
& \text { else return zero } \\
& \text { end if }
\end{aligned}
$$

\subsection{Simple Cart Classification Algorithm}

Simple Cart (Classification \& regression tree) is a classification technique that generates the binary decision tree. Since output is binary tree, it generates only two children. Entropy is used to choose the best splitting attribute. Simple Cart handles the missing data by ignoring that record. This algorithm is best for the training data. 


\subsection{J48 Classification Algorithm}

A decision tree is a predictive machine-learning model that decides the target value (dependent variable) of a new sample based on various attribute values of the available data. The internal nodes of a decision tree denote the different attributes; the branches between the nodes tell us the possible values that these attributes can have in the observed samples, while the terminal nodes tell us the final value (classification) of the dependent variable. The attribute that is to be predicted is known as the dependent variable, since its value depends upon, or is decided by, the values of all the other attributes. The other attributes, which help in predicting the value of the dependent variable, are known as the independent variables in the dataset. [12].

\subsection{ZeroR Classification algorithm}

ZeroR classifier predicts the majority of class in training data. It predicts the mean for numeric value \& mode for nominal class.

\subsection{Naive Bays Classification Algorithm}

Naïve Bays classification is based on Bays rule conditional probability. It makes use of all the attributes contained in the data, and analyses them individually as though they are equally important and independent of each other [12].

\subsection{Random Forest Classification Algorithm}

Random forest is a classifier that consists of many decision trees and outputs the class that is the mode of the classes output by individual trees. For many data sets, it produces a highly accurate classifier [13]. This algorithm handles the missing data $\&$ maintains the accuracy.

\subsection{Decision Table}

This is Class for building and using a simple decision table majority classifier (Ron Kohavi). In this algorithm, we have to set the number folds, display rule etc to get the proper result.

\section{EXPERIMENTAL RESULT}

Here we are considering the sample data extracted from Moodle database of a college after collection of data for course enrollment by student as shown in Table 1 . In this table, we consider 45 student \& 15 courses. Fifteen courses are C-programming (C), Visual Basic (VB), Active Server Pages (ASP), Computer Network (CN), Network Engineering (NE), Microprocessor (MP), Computer Organization (CO), Database Engineering (DBE), Advanced Database System (ADS), Operating System (OS), Distributed System (DS), Finite Automata System (FSA), Data Structure (DS-I), Software Engineering (SE), and Software Testing \& Quality assurance (STQA). In this table yes represent that the student is interested in that particular course \& no represent that student do not like that course.

Table 1: Sample Data from Moodle Database[11]

\begin{tabular}{|c|c|c|c|c|c|c|c|c|c|c|c|c|c|c|c|}
\hline $\begin{array}{l}\text { Courses } \rightarrow \\
\text { Roll_No } \downarrow\end{array}$ & $\mathrm{C}$ & VB & ASP & $\mathrm{CN}$ & $\mathrm{NE}$ & MP & $\mathrm{CO}$ & DBE & ADS & OS & DS & FSA & $\begin{array}{l}\text { DS- } \\
\text { I }\end{array}$ & SE & STQA \\
\hline 1 & yes & yes & yes & yes & yes & no & no & no & no & no & no & no & yes & no & no \\
\hline 2 & no & no & no & no & no & no & no & no & no & no & no & no & no & no & no \\
\hline 3 & yes & yes & yes & yes & yes & no & no & no & no & yes & yes & yes & yes & yes & yes \\
\hline 4 & no & no & no & yes & yes & no & yes & no & no & no & no & no & no & no & no \\
\hline 5 & yes & yes & yes & yes & yes & no & no & yes & no & yes & yes & no & yes & no & no \\
\hline 6 & yes & yes & yes & no & no & no & no & no & no & yes & no & no & yes & no & no \\
\hline 7 & no & no & no & yes & yes & yes & yes & no & no & no & no & no & no & yes & no \\
\hline 8 & no & no & no & no & no & no & no & yes & yes & yes & yes & no & yes & no & no \\
\hline 9 & no & no & no & yes & yes & yes & yes & no & no & no & no & yes & no & no & no \\
\hline 10 & yes & no & no & no & no & no & no & no & no & no & no & no & no & no & no \\
\hline 11 & yes & yes & yes & no & no & no & no & no & no & yes & yes & no & yes & no & no \\
\hline 12 & yes & yes & yes & yes & yes & no & no & no & no & no & no & no & no & no & no \\
\hline 13 & no & no & no & no & no & no & no & yes & yes & yes & yes & no & yes & yes & yes \\
\hline 14 & yes & yes & yes & yes & yes & no & no & no & no & yes & yes & no & no & no & no \\
\hline 15 & yes & yes & yes & no & no & no & no & no & no & no & no & no & yes & no & no \\
\hline 16 & no & no & no & yes & yes & no & no & yes & yes & yes & yes & no & yes & no & no \\
\hline 17 & yes & yes & yes & no & no & no & no & no & no & yes & yes & no & yes & yes & yes \\
\hline 18 & yes & yes & yes & no & no & no & no & no & no & no & no & no & no & no & no \\
\hline 19 & no & no & no & yes & yes & yes & yes & yes & yes & no & no & no & no & no & no \\
\hline 20 & yes & no & no & no & no & no & no & no & no & yes & yes & no & yes & yes & yes \\
\hline 21 & yes & no & yes & no & no & yes & yes & no & no & yes & yes & yes & no & no & no \\
\hline 22 & no & no & no & no & no & no & no & yes & yes & yes & yes & no & yes & no & no \\
\hline 23 & yes & yes & yes & yes & yes & yes & yes & no & no & yes & yes & no & yes & no & no \\
\hline 24 & yes & yes & yes & yes & yes & yes & yes & yes & yes & yes & yes & yes & yes & yes & yes \\
\hline 25 & no & yes & yes & no & no & yes & yes & yes & yes & yes & yes & no & no & no & no \\
\hline 26 & yes & yes & yes & no & no & no & no & no & no & yes & yes & no & yes & no & no \\
\hline 27 & yes & yes & yes & yes & yes & no & no & no & no & no & no & no & no & no & no \\
\hline 28 & no & no & no & yes & yes & no & no & no & no & yes & yes & no & yes & no & no \\
\hline 29 & no & no & no & no & no & yes & yes & yes & yes & no & no & no & no & no & no \\
\hline 30 & yes & yes & yes & yes & yes & no & no & no & no & no & no & no & no & yes & yes \\
\hline 31 & no & no & no & no & no & no & no & no & no & no & no & no & no & no & no \\
\hline 32 & yes & yes & yes & no & no & no & no & yes & yes & yes & yes & no & yes & no & no \\
\hline 33 & no & no & no & yes & yes & no & no & no & no & yes & yes & no & yes & no & no \\
\hline
\end{tabular}




\begin{tabular}{|l|l|l|l|l|l|l|l|l|l|l|l|l|l|l|l|}
\hline 34 & yes & yes & yes & no & no & no & no & no & no & no & no & no & no & no & no \\
\hline 35 & no & no & no & no & no & no & no & no & no & yes & yes & no & no & no & no \\
\hline 36 & no & no & no & yes & yes & no & no & no & no & no & no & no & yes & no & no \\
\hline 37 & yes & yes & yes & yes & yes & yes & yes & yes & yes & no & no & no & no & no & no \\
\hline 38 & no & no & no & no & no & no & no & no & no & yes & yes & yes & yes & yes & yes \\
\hline 39 & yes & yes & yes & yes & yes & yes & yes & yes & yes & yes & yes & yes & yes & yes & yes \\
\hline 40 & no & no & no & no & no & no & no & no & no & no & no & no & no & yes & yes \\
\hline 41 & yes & yes & yes & no & no & no & no & no & no & yes & yes & no & yes & no & no \\
\hline 42 & no & no & no & yes & yes & no & no & no & no & no & no & no & no & no & no \\
\hline 43 & no & no & no & no & no & no & no & no & no & yes & yes & no & yes & no & no \\
\hline 44 & no & no & no & no & no & no & no & no & no & no & no & no & no & no & yes \\
\hline 45 & no & no & no & no & no & no & no & no & no & no & no & no & no & no & no \\
\hline
\end{tabular}

In table 2, we are considering only those courses from table 1 for which the classification algorithm classifies this course as "yes". For remaining courses, the classification algorithm gives more percentage of "no" compare to the percentage of "yes". Table 3 gives the simulation errors that occurred in each classification algorithm.

From table 2, we can observe that ADTree has highest percentage of correctly classified instance \& lowest percentage of incorrectly classified instances. ZeroR classification algorithm has lowest percentage of correctly classified instances \& highest percentage of incorrectly classified instances. Naive Bays \& Decision Table has the same percentage for correctly \& incorrectly classified instances. Simple Cart, J48, \& Random Forest classification algorithm has $91.66 \%, 93.33 \%$ \& $87.22 \%$ correctly classified instances respectively \& $8.44 \%, 6.77 \%$, and $12.88 \%$ incorrectly classified instances. Ascending order of classification algorithm considering the classification accuracy into account is ADTree, J48, Naive Bays, Decision Table, Simple Cart, Random Forest, and ZeroR. So we consider the ADTree as classification algorithm for Course Recommender System as classification accuracy for ADTree is highest.

Table 2: Result using different classification algorithm

\begin{tabular}{|c|c|c|c|c|c|c|c|c|}
\hline \multicolumn{2}{|c|}{$\begin{array}{l}\text { Classification algorithm } \\
\rightarrow \\
\text { Courses } \downarrow\end{array}$} & $\begin{array}{l}\text { Naive } \\
\text { Bays }\end{array}$ & $\begin{array}{l}\text { Simple } \\
\text { Cart }\end{array}$ & ZeroR & ADTree & $\begin{array}{l}\text { Decision } \\
\text { Table }\end{array}$ & $\mathrm{J} 48$ & $\begin{array}{l}\text { Random } \\
\text { Forest }\end{array}$ \\
\hline \multirow{2}{*}{$\begin{array}{c}\text { C- } \\
\text { programming }\end{array}$} & $\begin{array}{l}\text { Correctly } \\
\text { classified } \\
\text { instance }\end{array}$ & 42 & 41 & 23 & 43 & 41 & 42 & 41 \\
\hline & $\begin{array}{l}\text { Incorrectly } \\
\text { classified } \\
\text { instance }\end{array}$ & 3 & 4 & 22 & 2 & 4 & 3 & 4 \\
\hline \multirow{2}{*}{$\begin{array}{l}\text { Operating } \\
\text { System }\end{array}$} & $\begin{array}{l}\text { Correctly } \\
\text { classified } \\
\text { instance }\end{array}$ & 44 & 44 & 24 & 44 & 44 & 44 & 43 \\
\hline & $\begin{array}{l}\text { Incorrectly } \\
\text { classified } \\
\text { instance }\end{array}$ & 1 & 1 & 21 & 1 & 1 & 1 & 2 \\
\hline \multirow{2}{*}{$\begin{array}{l}\text { Distributed } \\
\text { System }\end{array}$} & $\begin{array}{l}\text { Correctly } \\
\text { classified } \\
\text { instance }\end{array}$ & 43 & 44 & 23 & 44 & 44 & 44 & 43 \\
\hline & $\begin{array}{l}\text { Incorrectly } \\
\text { classified } \\
\text { instance }\end{array}$ & 2 & 1 & 22 & 1 & 1 & 1 & 2 \\
\hline \multirow{2}{*}{$\begin{array}{c}\text { Data } \\
\text { Structure-I }\end{array}$} & $\begin{array}{l}\text { Correctly } \\
\text { classified } \\
\text { instance }\end{array}$ & 38 & 36 & 23 & 40 & 38 & 38 & 30 \\
\hline & $\begin{array}{l}\text { Incorrectly } \\
\text { classified } \\
\text { instance }\end{array}$ & 7 & 9 & 22 & 5 & 7 & 7 & 15 \\
\hline \multirow{2}{*}{ Overall result } & $\begin{array}{l}\text { Correctly } \\
\text { classified } \\
\text { instance }\end{array}$ & $\begin{array}{c}167 \\
(92.77 \%)\end{array}$ & $\begin{array}{c}165 \\
(91.66 \%)\end{array}$ & $\begin{array}{c}93 \\
(51.66 \%)\end{array}$ & $\begin{array}{c}172 \\
(95.55 \%)\end{array}$ & $\begin{array}{c}167 \\
(92.77 \%)\end{array}$ & $\begin{array}{c}168 \\
(93.33 \%)\end{array}$ & $\begin{array}{c}157 \\
(\mathbf{8 7 . 2 2})\end{array}$ \\
\hline & $\begin{array}{l}\text { Incorrectly } \\
\text { classified } \\
\text { instance }\end{array}$ & $\begin{array}{c}13 \\
(7.23 \%)\end{array}$ & $\begin{array}{c}15 \\
(8.44 \%)\end{array}$ & $\begin{array}{c}87 \\
(48.44 \%)\end{array}$ & $\begin{array}{c}9 \\
(4.45 \%)\end{array}$ & $\begin{array}{c}13 \\
(7.33 \%)\end{array}$ & $\begin{array}{c}12 \\
(6.77 \%)\end{array}$ & $\begin{array}{c}23 \\
(12.88 \%)\end{array}$ \\
\hline
\end{tabular}


Table 3: Simulation Errors in classification algorithm

\begin{tabular}{|c|c|c|c|c|c|c|c|c|}
\hline \multicolumn{2}{|c|}{$\begin{array}{c}\text { Classification algorithm } \rightarrow \\
\text { Courses } \downarrow\end{array}$} & $\begin{array}{l}\text { Naive } \\
\text { Bays }\end{array}$ & $\begin{array}{l}\text { Simple } \\
\text { Cart }\end{array}$ & ZeroR & ADTree & $\begin{array}{c}\text { Decision } \\
\text { Table }\end{array}$ & $\mathrm{J} 48$ & $\begin{array}{c}\text { Random } \\
\text { Forest }\end{array}$ \\
\hline \multirow{4}{*}{$\begin{array}{c}\text { C- } \\
\text { programmi } \\
\text { ng }\end{array}$} & Mean absolute error & 0.1066 & 0.1478 & 0.5011 & 0.2168 & 0.1478 & 0.1478 & 0.1799 \\
\hline & $\begin{array}{l}\text { Root mean squared } \\
\text { error }\end{array}$ & 0.2708 & 0.2997 & 0.5012 & 0.3274 & 0.2997 & 0.2997 & 0.319 \\
\hline & $\begin{array}{l}\text { Relative absolute } \\
\text { error }\end{array}$ & $21.2766 \%$ & $29.5017 \%$ & $100 \%$ & $43.2628 \%$ & $29.5017 \%$ & $\begin{array}{c}29.5017 \\
\%\end{array}$ & $35.9018 \%$ \\
\hline & $\begin{array}{l}\text { Root relative squared } \\
\text { error }\end{array}$ & $54.0313 \%$ & $59.7928 \%$ & $100 \%$ & $65.3139 \%$ & $59.7928 \%$ & $\begin{array}{c}59.7928 \\
\% \\
\end{array}$ & $63.6473 \%$ \\
\hline \multirow{4}{*}{$\begin{array}{l}\text { Operating } \\
\text { System }\end{array}$} & Mean absolute error & 0.0937 & 0.0435 & 0.4989 & 0.0791 & 0.0435 & 0.0435 & 0.1207 \\
\hline & $\begin{array}{l}\text { Root mean squared } \\
\text { error }\end{array}$ & 0.2036 & 0.1526 & 0.5 & 0.1929 & 0.1526 & 0.1526 & 0.2287 \\
\hline & $\begin{array}{l}\text { Relative absolute } \\
\text { error }\end{array}$ & $18.7894 \%$ & $8.7202 \%$ & $100 \%$ & $15.8582 \%$ & $8.7202 \%$ & $8.7202 \%$ & $24.1994 \%$ \\
\hline & $\begin{array}{l}\text { Root relative squared } \\
\text { error }\end{array}$ & $40.7211 \%$ & $30.5267 \%$ & $100 \%$ & $38.5807 \%$ & $30.5267 \%$ & $\begin{array}{c}30.5267 \\
\%\end{array}$ & $45.7326 \%$ \\
\hline \multirow{4}{*}{$\begin{array}{l}\text { Distributed } \\
\text { System }\end{array}$} & Mean absolute error & 0.1056 & 0.0427 & 0.5011 & 0.1166 & 0.0427 & 0.0427 & 0.1456 \\
\hline & $\begin{array}{l}\text { Root mean squared } \\
\text { error }\end{array}$ & 0.2202 & 0.1522 & 0.5012 & 0.2052 & 0.1522 & 0.1522 & 0.2441 \\
\hline & $\begin{array}{l}\text { Relative absolute } \\
\text { error }\end{array}$ & $21.0675 \%$ & $8.5245 \%$ & $100 \%$ & $23.2764 \%$ & $8.5245 \%$ & $8.5245 \%$ & $29.0618 \%$ \\
\hline & $\begin{array}{l}\text { Root relative squared } \\
\text { error }\end{array}$ & $43.93 \%$ & $30.3662 \%$ & $100 \%$ & $40.9439 \%$ & $30.3662 \%$ & $\begin{array}{c}30.3662 \\
\%\end{array}$ & $48.6894 \%$ \\
\hline \multirow{4}{*}{$\begin{array}{c}\text { Data } \\
\text { Structure-I }\end{array}$} & Mean absolute error & 0.2364 & 0.286 & 0.5011 & 0.3714 & 0.2734 & 0.2825 & 0.3286 \\
\hline & $\begin{array}{l}\text { Root mean squared } \\
\text { error }\end{array}$ & 0.4071 & 0.3959 & 0.5012 & 0.4427 & 0.4127 & 0.4026 & 0.4863 \\
\hline & $\begin{array}{ll}\begin{array}{l}\text { Relative } \\
\text { error }\end{array} & \text { absolute } \\
\end{array}$ & $47.1806 \%$ & $57.0873 \%$ & $100 \%$ & $74.1151 \%$ & $54.5589 \%$ & $\begin{array}{c}56.3758 \\
\%\end{array}$ & $65.5755 \%$ \\
\hline & $\begin{array}{l}\text { Root relative squared } \\
\text { error }\end{array}$ & $81.2174 \%$ & $78.9805 \%$ & $100 \%$ & $88.3127 \%$ & $82.3421 \%$ & $\begin{array}{c}80.3289 \\
\%\end{array}$ & $97.0259 \%$ \\
\hline
\end{tabular}

The graph for correctly classified instances is shown in figure 2 while the graph for incorrectly classified instance is shown in figure 3 . From these two graphs, we can observe that ADTree classification algorithm is best for Course Recommender System.

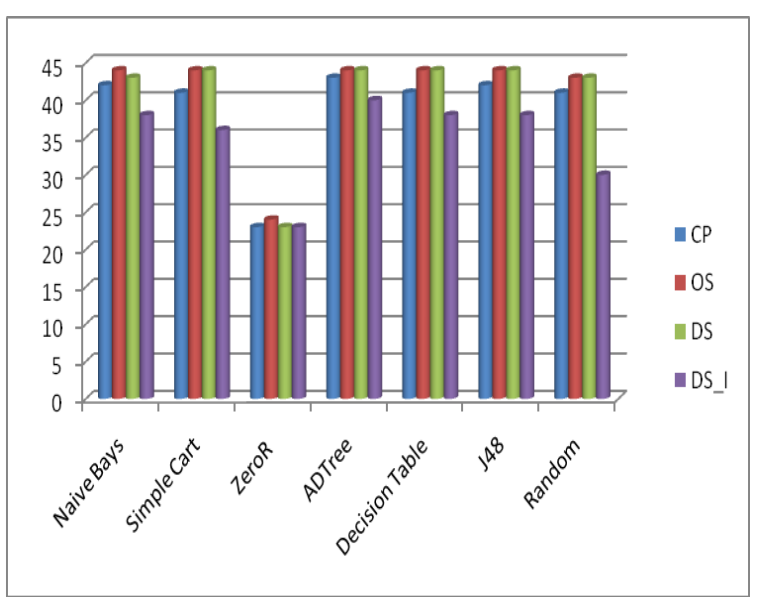

Figure 2: Graph for correctly classified instance in various classification algorithms
In this graphs $\mathrm{X}$-axis represent the seven classification algorithm while Y-axis represents correctly \& incorrectly classified instances.

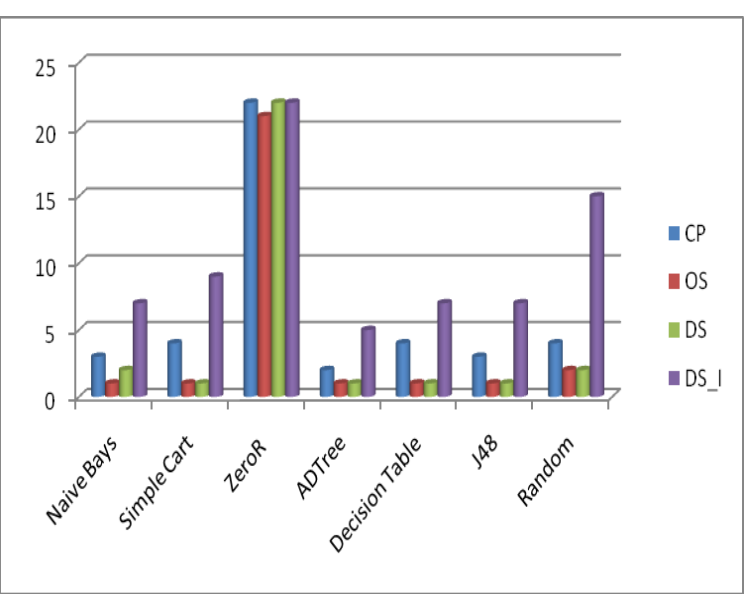

Figure 3: Graph for incorrectly classified instance in various classification algorithms 
Figure 4 shows the graph for comparison of these seven classification algorithm. In this graph, $\mathrm{X}$-axis represents courses considered for comparison of classification algorithm \& Y-axis represent the correctly classified \& incorrectly classified instances. The result of application after application of ADTree classification algorithm to table 1 is shown in table 4 .

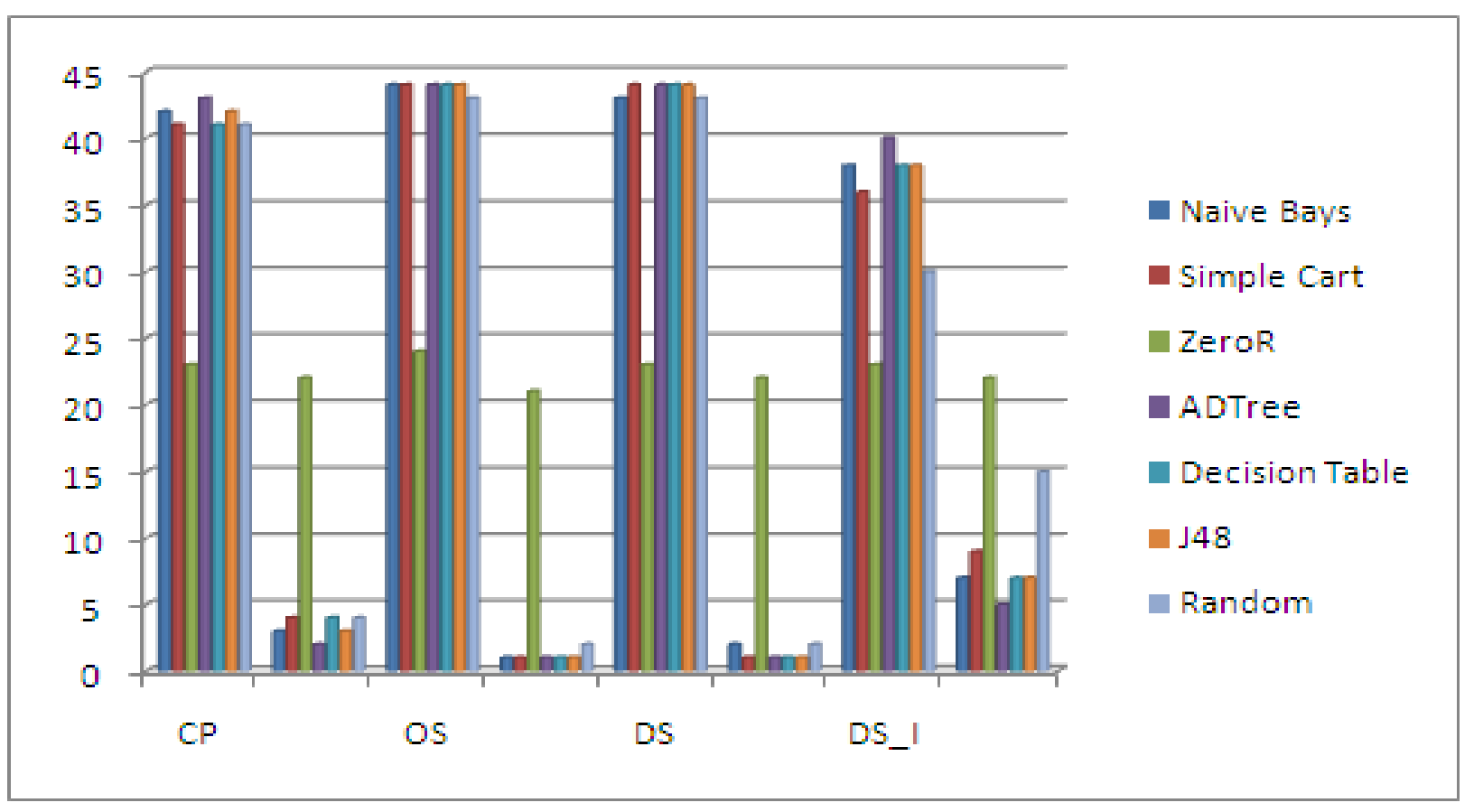

Figure 4: Comparison of classification algorithms in Course Recommendation System

Table 4: Table after application of classification algorithm-ADTree

\begin{tabular}{|r|l|l|l|l|l|l|l|l|l|l|l|l|l|l|l|}
\hline $\begin{array}{l}\text { Courses } \\
\rightarrow\end{array}$ \\
Roll_No
\end{tabular}

After classifying the data, we apply the Apriori association rule to get the best combination of courses. Before preprocessing of data, we got the association rule containing "no" only which is shown in first row of table 5.

As we are recommending the course, we preprocess the data. The result after preprocessing of data is shown in second row of table 5. Now the association rule contains only "yes". The result of applying the Apriori association rule on classified data is shown in third row of table 5. 
Table 5: Result for Course Recommender System [14]

\begin{tabular}{|c|c|c|}
\hline $\begin{array}{c}\text { Course } \\
\text { considered }\end{array}$ & $\begin{array}{l}\text { Parameter } \\
\text { Considered }\end{array}$ & Results \\
\hline \multicolumn{3}{|c|}{$\begin{array}{c}\text { Result of Apriori Association Rule before preprocessing \& application of combination of Classification \& } \\
\text { Association Rule }\end{array}$} \\
\hline $\begin{array}{lr}\text { C, } & \text { VB, } \\
\text { ASP, } & \text { CN, } \\
\text { NE, } & \text { MP, } \\
\text { CO, } & \text { DBE, } \\
\text { ADS, OS, } \\
\text { DS, } & \text { FSA, } \\
\text { DS-I, } & \text { SE, } \\
\text { STQA }\end{array}$ & $\begin{array}{l}\text { Minimum } \\
\text { support: } 0.7 \\
\text { Minimum } \\
\text { metric } \\
\text { <confidence>: } \\
0.9\end{array}$ & $\begin{array}{l}\text { Best rules found: } \\
\text { 1. Computer_Organization }=\text { no } \rightarrow \text { Microprocessor }=\text { no } \\
\text { 2.Database_Engg=no } \rightarrow \text { Advanced_Database_System }=\text { no } \\
\text { 3. Computer_Organization }=\text { no Finite_State_Automata }=\text { no } \rightarrow \text { Microprocessor=no } \\
\text { 4. Microprocessor }=\text { no } \rightarrow \text { Computer_Organization }=\text { no } \\
\text { 5.Software_Testing_\&_Quality_Assurance=no } \rightarrow \text { Software_Engg=no } \\
\text { 6. Software_Engg =no } \rightarrow \text { Software_Testing_\&_Quality_Assurance }=\text { no } \\
\text { 7. Advanced_Database_System }=\text { no } \rightarrow \text { Database_Engg }=\text { no } \\
\text { 8. Microprocessor }=\text { no Finite_State_Automata }=\text { no } \rightarrow \text { Computer_Organization }=\text { no } \\
\text { 9. Finite_State_Automata }=\text { no Software_Testing_\&_Quality_Assurance }=\text { no } \rightarrow \\
\text { Software_Engg =no } \\
\text { 10. Finite_State_Automata }=\text { no Software_Engg }=\text { no } \rightarrow \\
\text { Software_Testing_\&_Quality_Assurance =no }\end{array}$ \\
\hline \multicolumn{3}{|c|}{$\begin{array}{c}\text { Result of Apriori Association Rule after preprocessing \& before application of combination of Classification \& } \\
\text { Association Rule }\end{array}$} \\
\hline \multirow[t]{2}{*}{$\begin{array}{l}\text { C, VB, } \\
\text { ASP, CN, } \\
\text { NE, OS, } \\
\text { DS, DS-I }\end{array}$} & $\begin{array}{l}\text { Minimum } \\
\text { support: } 0.5 \\
\text { Minimum } \\
\text { metric } \\
\text { <confidence>: } \\
0.9\end{array}$ & $\begin{array}{l}\text { Best rules found: } \\
\text { 1. Distributed_System }=\text { yes } \rightarrow \text { Operating_System }=\text { yes } \\
\text { 2. Visual_Basic=yes } \rightarrow \text { Active_Server_Pages=yes } \\
\text { 3. Network_Engg=yes } \rightarrow \text { Computer_Network=yes } \\
\text { 4. Computer_Network }=\text { yes } \rightarrow \text { Network_Engg =yes } \\
\text { 5. C_Programming=yes Visual_Basic =yes } \rightarrow \text { Active_Server_Pages =yes } \\
\text { 6. Distributed_System }=\text { yes Data_Sstructure_I=yes } \rightarrow \text { Operating_System }=\text { yes } \\
\text { 7. Operating_System }=\text { yes } \rightarrow \text { Distributed_System }=\text { yes } \\
\text { 8. Active_Server_Pages }=\text { yes } \rightarrow \text { C_Programming }=\text { yes } \\
\text { 9. C_Programming =yes } \rightarrow \text { Active_Server_Pages =yes } \\
\text { 10. Active_Server_Pages }=\text { yes } \rightarrow \text { Visual_Basic }=\text { yes }\end{array}$ \\
\hline & $\begin{array}{l}\text { Minimum } \\
\text { support: } 0.6 \\
\text { Minimum } \\
\text { metric } \\
\text { <confidence>: } \\
0.9\end{array}$ & $\begin{array}{l}\text { Best rules found: } \\
\text { 1. Distributed_System }=\text { yes } \rightarrow \text { Operating_System }=\text { yes } \\
\text { 2. Operating_System }=\text { yes } \rightarrow \text { Distributed_System }=\text { yes }\end{array}$ \\
\hline \multicolumn{3}{|c|}{ After Application of Classification algorithm-ADTree \& Association Rule-Apriori Association Rule } \\
\hline $\begin{array}{l}\text { C, VB, } \\
\text { ASP, CN, } \\
\text { NE, MP, } \\
\text { CO, DBE, } \\
\text { ADS, OS, } \\
\text { DS, FSA, } \\
\text { DS-I, SE, } \\
\text { STQA }\end{array}$ & $\begin{array}{l}\text { Minimum } \\
\text { support: } 0.95 \\
\text { Minimum } \\
\text { metric } \\
\text { <confidence> } \\
: 0.9\end{array}$ & $\begin{array}{l}\text { Best rules found: } \\
\text { 1. Operating_System }=\text { yes } \rightarrow \text { C_Programming }=\text { yes } \\
\text { 2. C_Programming }=\text { yes } \rightarrow \text { Operating_System }=\text { yes } \\
\text { 3. Distributed_System }=\text { yes } \rightarrow \text { C_Programming }=\text { yes } \\
\text { 4. C_Programming }=\text { yes } \rightarrow \text { Distributed_System }=\text { yes } \\
\text { 5. Data_Sstructure_I =yes } \rightarrow \text { C_Programming }=\text { yes } \\
\text { 6. C_Programming }=\text { yes } \rightarrow \text { Data_Sstructure_I }=\text { yes } \\
\text { 7. Distributed_System }=\text { yes } \rightarrow \text { Operating_System }=\text { yes } \\
\text { 8. Operating_System }=\text { yes } \rightarrow \text { Distributed_System }=\text { yes } \\
\text { 9. Data_Sstructure_I }=\text { yes } \rightarrow \text { Operating_System }=\text { yes } \\
\text { 10. Operating_System }=\text { yes } \rightarrow \text { Data_Sstructure_I =yes }\end{array}$ \\
\hline
\end{tabular}




\section{CONCLUSION AND FUTURE WORK}

Here in this paper we compare the seven classification algorithm to choose the best classification algorithm for recommending the course to student based on various student choices. Theses seven classification algorithms, we consider for comparison, are ADTree, Simple Cart, J48, ZeroR, Naive Bays, Decision Table \& Random Forest Classification Algorithm. We use the open source data mining tool Weka to check the result. We find that ADTree classification algorithm works better for this Course Recommender System as incorrectly classified instance for this algorithms are less than other five classification algorithms. Future works include the combination of other data mining algorithms to recommend the course to the student from the data obtained from the Moodle course of the college.

\section{REFERENCES}

[1] Hu H., Li J., Plank A., Wang H. and Daggard G., "A Comparative Study of Classification Methods for Microarray Data Analysis", In Proc. Fifth Australasian Data Mining Conference, Sydney, Australia (2006).

[2] Abdelghani Bellaachia, Erhan Guven, "Predicting Breast Cancer Survivability Using Data Mining Techniques" accessed from http://www.siam.org/meetings/sdm06/workproceed/S cientific\%20Datasets/bellaachia.pdf on 05-03-2012

[3] My Chau Tu, Dongil Shin, Dongkyoo Shin, "A Comparative Study of Medical Data Classification Methods Based on Decision Tree and Bagging Algorithms", dasc, pp.183-187, 2009 Eighth IEEE International Conference on Dependable, Autonomic and Secure Computing, 2009.

[4] Aman Kumar Sharma, Suruchi Sahni:"A Comparative Study of Classification Algorithms for Spam Email Data Analysis" in International Journal on Computer Science and Engineering (IJCSE)

[5] Rich Caruana Alexandru Niculescu-Mizil," An Empirical Comparison of Supervised Learning Algorithms" ICML '06 Proceedings of the 23rd international conference on Machine learning. ISBN:1-59593-383-2 doi>10.1145/1143844.1143865

[6] ERIC BAUER, RON KOHAVI," An Empirical Comparison of Voting Classification Algorithms: Bagging, Boosting, and Variants" Machine Learning, vv, $1\{38$ (1998) Kluwer Academic Publishers, Boston. Manufactured in The Netherlands.
[7] TJEN-SIEN LIM, WEI-YIN LOH, YU-SHAN SHIH," A Comparison of Prediction Accuracy, Complexity, and Training Time of Thirty-three Old and New Classi_cation Algorithms" Machine Learning, 40, 203\{229 (2000) Kluwer Academic Publishers, Boston. Manufactured in The Netherlands.

[8] Weka (2007). http://www.cs.waikato.ac.nz/ml/weka/.

[9] "Data Mining Introductory and Advanced Topics" by Margaret H. Dunham

[10] Sunita B Aher and Lobo L.M.R.J.. Data Mining in Educational System using WEKA. IJCA Proceedings on International Conference on Emerging Technology Trends (ICETT) (3):20-25, 2011. Published by Foundation of Computer Science, New York, USA (ISBN: 978-93-80864-71-13)

[11] Sunita B Aher and Lobo L.M.R.J. Article: A Framework for Recommendation of courses in Elearning System. International Journal of Computer Applications 35(4):21-28, December 2011. Published by Foundation of Computer Science, New York, USA ISSN 0975 - $\mathbf{8 8 8 7}$ Digital Library URI: http://www.ijcaonline.org/archives/volume35/number 4/4389-6091.

[12] http://www.d.umn.edu/ padhy005/Chapter5.html accessed on 30-01-2012

[13] http://en.wikipedia.org/wiki/Random_forest accessed on 30-01-2012

[14] Sunita B Aher and Lobo L.M.R.J. Article: Mining Association Rule in Classified Data for Course Recommender System in E-Learning. International Journal of Computer Applications 39(7):1-7, February 2012. Published by Foundation of Computer Science, New York, USA. ISSN 0975 - 8887 Digital Library URI:

http://www.ijcaonline.org/archives/volume39/number 7/4829-7086

[15] Sunita B Aher and Lobo L.M.R.J.,'Data Preparation Strategy in E-Learning System using Association Rule Algorithm" selected in International Journal of Computer Applications. Published by Foundation of Computer Science, New York, USA. ISSN 0975 8887

[16] http://en.wikipedia.org/wiki/Alternating_decision_tree accessed on dat 02-02-2012 